\title{
L'espace de la politique forestière en question
}

The forestry policy space in question

\section{Robin Degron}

\section{(2) OpenEdition}

\section{Journals}

Édition électronique

URL : http://journals.openedition.org/rge/1904

DOI : $10.4000 /$ rge. 1904

ISSN : 2108-6478

\section{Éditeur}

Association des géographes de l'Est

\section{Édition imprimée}

Date de publication : 1 janvier 2009

ISSN : 0035-3213

\section{Référence électronique}

Robin Degron, "L'espace de la politique forestière en question », Revue Géographique de l'Est [En ligne], vol. 49 / 2-3 | 2009, mis en ligne le 04 octobre 2010, consulté le 08 septembre 2020. URL : http:// journals.openedition.org/rge/1904 ; DOI : https://doi.org/10.4000/rge.1904

Ce document a été généré automatiquement le 8 septembre 2020

Tous droits réservés 


\title{
L'espace de la politique forestière en question
}

\author{
The forestry policy space in question
}

Robin Degron

\section{Introduction}

Objet des sciences naturelles et des sciences humaines, la forêt est un terrain d'investigation privilégié pour le géographe ${ }^{1}$. Celui-ci doit mobiliser à la fois les compétences de l'écologue, de l'économiste, de l'historien, du sociologue, du politologue et du juriste pour tenter de comprendre la complexité des interactions tissées sur le temps long entre l'homme et l'espace forestier ${ }^{2}$. La recherche sur l'espace forestier s'élargit nécessairement pour toucher au cadre normatif et institutionnel, à l'ensemble des règles et des structures qui reflètent les attentes d'une société et les moyens dont elle dispose pour gérer les forêts. La politique forestière et ses instruments deviennent, par eux mêmes, objets d'analyse géographique.

$2 \mathrm{Vu}$ sous cet angle et replacé dans le contexte européen, le cas français interroge : il appelle un questionnement sur l'espace de référence pour la définition d'une stratégie globale intégrant les fonctions économiques, environnementales et sociales de la forêt. Héritière d'une longue histoire administrative centralisatrice (Buttoud, 1983; Collectif, 1987), la politique forestière nationale semble peiner à s'adapter aux cadres nouveaux posés par la construction européenne. Après la définition des stratégies de Lisbonne et de Göteborg, le rôle de l'Union en matière sylvicole devrait pourtant être naturellement rehaussé. Soumises à la volonté de l'Etat central, des services déconcentrés du ministère de l'agriculture et de l'office national des forêts, les orientations de la politique forestière semblent par ailleurs faire trop peu de place aux collectivités territoriales, singulièrement aux conseils régionaux dont les attributions dans le domaine de l'aménagement du territoire et du développement économique vont pourtant croissant. En adoptant la grille de lecture offerte par le concept de géoalliance (Degron, 2007 et 2008), nous serions tentés de dire que les modes de fonctionnement et 
les structures de la politique forestière française paraissent aujourd'hui "décalées " par rapport aux barreaux de la nouvelle échelle des pouvoirs économiques et environnementaux qui relie l'Union européenne et les régions. L'association durable, spatialement, juridiquement et financièrement structurée entre l'Europe et ses régions pousse naturellement à repenser les cadres géographiques de référence de la politique forestière hexagonale.

De la mise en évidence de l'écart entre le cadre conceptuel prospectif d'une politique forestière européenne déclinée en région et le système actuel d'une politique nationale déconcentrée découle la nécessité d'un examen fin et actualisé des jeux d'acteurs et des évolutions institutionnelles. Comme la théorie de la géoalliance le laisser supposer, l'Union s'affirme comme le nouveau décideur en matière forestière et tend à remettre en cause, depuis peu, la place jusque là stratégique réservée à l'Etat forestier. Cette évolution est encore mal perçue en France ( $1^{\text {ère }}$ partie). Dans le même temps, les régions françaises renforcent leur autorité et leurs moyens d'action dans les domaines économique et de l'aménagement du territoire. De façon encore très variable, les conseils régionaux commencent à s'intéresser au secteur forestier en tant que tel. De fait, la récente politique forestière définie en Corse, dans un cadre géopolitique jusque là considéré comme singulier, interroge quant à la possibilité de généraliser un modèle forestier décentralisé sur l'ensemble du territoire ( $2^{\text {nde }}$ partie).

\section{I - L'Union européenne s'affirme comme le nouveau stratège de la politique forestière parmi les Etats- membres}

\section{A - Le plan d'action forestier européen 2007-2011 marque un tournant dans l'affirmation de l'Europe forestière}

Une politique publique se construit par étapes successives, avec des phases d'accélération plus ou moins sensibles selon l'évolution des structures institutionnelles qui la portent et les variations conjoncturelles auxquelles les pouvoirs publiques européens, nationaux ou locaux sont soumis. La politique forestière européenne n'échappe pas à la règle. Son cours s'accélère au milieu des années 90 pour aboutir aujourd'hui à la mise en place d'un plan d'action forestier européen pour la période 2007-2011.

\section{Les prémisses de la politique forestière de l'Union européenne}

5 Dès le XIXème siècle, le développement de l'appareil statistique ou plus généralement de connaissance des forêts est le signe avant-coureur d'une volonté politique de prise en main (Degron, 1999). Ainsi, les rapports De Forcade et Faré adressés, en 1860 au ministre des finances ${ }^{3}$, marquent-ils le début de la grande vague des conversions en futaie régulière des forêts françaises (Degron, 1998). Le rapport sur «L'Europe de la forêt» commandée par le Parlement européen (Eurofor, 1994) correspond à l'émergence d'une vision européenne d'avenir forestier. Certes, avant cette date, la Communauté économique européenne soutenait déjà des actions en faveur du développement forestier. Cependant, selon les termes du rapport Eurofor, «L'absence de bilan financier ou technique, voire même d'information de synthèse actualisée et accessible, sur 
les actions passées de la Communauté en matière forestière en empêche toute évaluation quantifiée argumentée. ... Ces difficultés manifestent en elles-mêmes l'insuffisance de coordination et d'organisation de l'Union européenne en matière forestière. »

Développée au moment où se prépare l'élargissement de l'Union à la Suède, à la Finlande et à l'Autriche - tous trois grands pays forestiers ${ }^{4}$-, le rapport sur «L'Europe des forêts " offre non seulement une vision panoramique des forêts d'Europe mais propose les lignes directrices d'une stratégie forestière européenne. Il insiste sur le concept de multifonctionnalité, principe qui semble compatible avec l'ensemble des traditions sylvicoles nationales. Prudentes quant à la possibilité de voir émerger une nouvelle politique sectorielle européenne, les conclusions du rapport tendent plutôt à promouvoir une coordination souple des actions de l'Union en matière forestière et souligne l'importance de dédier des fonds à la réalisation des objectifs assignés à la gestion sylvicole. Rédigé sous la direction de l'office national des forêts, qui a su se placer comme le principal conseil du Parlement européen, le rapport de 1994 fait assez peu de place à la question régionale. Polysémique, le terme de région sert tour à tour d'enveloppe à la notion de région naturelle, de circonscription administrative de l'Etat ou d'entité géopolitique adossée à une assemblée élue. A titre d'exemple, la présentation de la forêt allemande par type de propriétaire fait l'amalgame entre les forêts fédérales et les forêts des Länder. Le texte ne précise pas que depuis la réunification de 1990, le Bund a transféré aux Länder la propriété et la gestion de l'essentiel du patrimoine forestier étatique ${ }^{5}$. Alors que le rapport fait une large place à la dimension technique du fait forestier, le poids économique et social du secteur forêtbois demeure par ailleurs mal évalué (Arnould, Hotyat, Simon, 1997). Au final, le document apparait, malgré la somme considérable d'informations collectées, comme étant encore assez incomplet sur le plan socio-économique et flou sur le plan institutionnel.

7 En cohérence avec la conclusion proposée par le rapport Eurofor, l'Union se dote en 1998 d'une première stratégie forestière de portée limitée. Sans remettre en cause la compétence première des Etats-membres dans la définition et la conduite des politiques forestières au sein de l'Union, la stratégie de 1998 offre un cadre de référence intellectuel commun aux forestiers d'Europe. L'apport que représente le budget européen à la mise en œuvre des lignes directrices esquissées est, il est vrai, modeste : l'Union ne peut guère mobiliser qu'une faible partie des fonds consacrés au second pilier de la Politique agricole commune (PAC) - le pilier du développement rural - luimême plafonné à hauteur de $10 \%$ des dépenses consenties pour la PAC, soit environ 4,3 Mds $€$ sur un total de 40,5 Mds $€$ pour l'année 2000. Les moyens alloués par le Programme d'intérêt communautaire (PIC) $\mathrm{Life}^{6}$ en matière de protection de la biodiversité et le Programme cadre de la recherche développement (PCRD) ne peuvent que contribuer marginalement au financement de la stratégie forestière européenne émergente.

\section{Le plan d'action forestier (2007-2011) : I'Union force de proposition et coordinatrice d'une nouvelle politique sectorielle européenne}

8 Le rapport sur la mise en oeuvre de la stratégie forestière de l'Union, présenté par la Commission européenne en 2005 souligne l'importance des enjeux économiques, environnementaux et sociaux que représente le secteur forestier ${ }^{7}$. Il franchit un cap dans le mouvement d'affirmation d'une politique forestière européenne. En insistant 
sur l'évolution rapide du contexte international et européen depuis 1998, le rapport pointe plusieurs arguments en faveur d'un approfondissement de la construction de l'Europe des forêts.

$\mathrm{Au}$ niveau international, la Commission replace la question forestière dans la perspective du Sommet mondial du développement durable de Johannesburg (2002) et rappelle l'unité de vue paneuropéenne sur la gestion des forêts telle qu'elle ressort des débats de la Conférence sur la protection des forêts d'Europe, tenue à Vienne, en 2003, sous l'égide de la Commission économique pour l'Europe des Nations Unies (CEE-NU).

$\mathrm{Au}$ niveau européen, le rapport de la Commission analyse en premier lieu les conséquences à tirer, d'une part de l'évolution tendancielle de la PAC et de l'importance croissante du pilier dédié au développement rural et, d'autre part, des ambitions affichés par le $6^{\text {ème }}$ Plan d'action environnementale communautaire. En second lieu, la Commission insiste lourdement sur la nécessité de prendre en compte les stratégies de Lisbonne et de Göteborg visant l'essor d'une économie performante, fondée sur l'innovation, et faisant une large place à la préservation des ressources et à la protection de l'environnement. Le tout est vu comme un facteur clef de développement économique durable. La question de l'indépendance énergétique de l'Union et celle, concomitante, de la lutte contre le changement climatique présente naturellement un intérêt particulier pour la Commission. Enfin, en filigrane de l'ensemble du rapport de 2005, l'élargissement massif de l'Union aux pays de l'Europe centrale et orientale est sans doute une source d'explication décisive au regain d'intérêt pour la forêt. Déjà, l'élargissement de 1996 avait sensiblement renforcé le taux de boisement communautaire. Après celui de 2004, le secteur forêt-bois s'impose, à l'évidence, comme un grand chantier d'intérêt communautaire (cf. Graphe A).

Graphe A - L'élargissement de l'Union européenne et l'évolution du taux de boisement communautaire (données FAO, 2006)

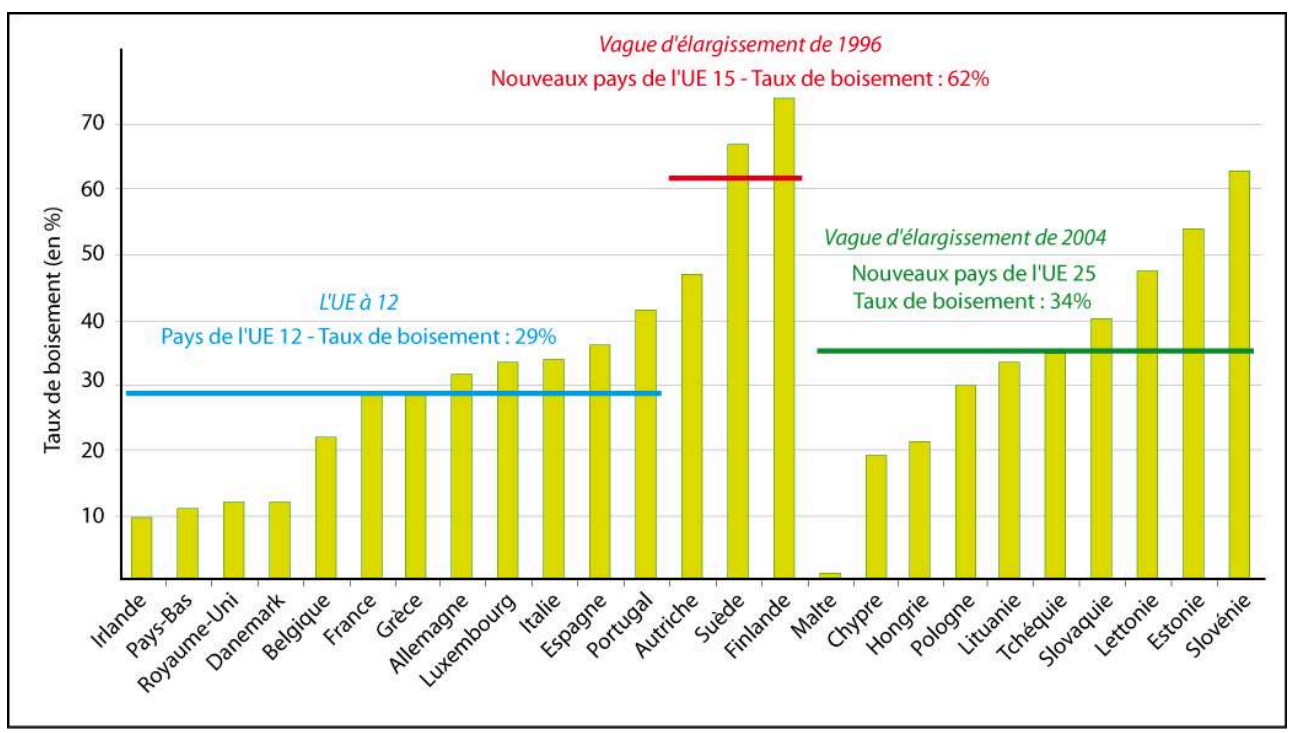

11 Conformément aux ambitions affichées en 2005, la communication de la Commission au Conseil et au Parlement européen, datée de juin 2006, prône une véritable politique forestière européenne ${ }^{8}$. Tout en rappelant le principe fondamental de multifonctionnalité, l'Union donne corps à un plan d'action forestier opérationnel qui s'appuie sur des ressources financières diversifiées et globalement plus importantes 
qu'à la fin des années 90. Outre l'apport du PIC Life et du PCRD, les moyens susceptibles d'appuyer la nouvelle politique sectorielle communautaire s'étendent à mesure que l'importance du second pilier de la PAC croît, via l'instauration du FEADER 9 . Distinct du FEOGA $^{10}$, ce nouveau fonds représente une augmentation sensible des moyens de l'Union au bénéfice du développement rural: avec 12,4 Mds $€$ pour 2007, le FEADER représente l'équivalent de $29 \%$ des fonds encore affectés au volet "production » de la PAC (42,7 Mds $€$ en 2007), soit environ trois fois plus que ce qu'apportait le $2^{\text {nd }}$ pilier de la PAC en 2000. En outre, fait nouveau dans l'approche européenne de la question forestière, les projets sylvicoles participant à la réalisation des objectifs des stratégies de Lisbonne et Göteborg peuvent s'appuyer sur le fond structurel de droit commun qu'est le FEDER ${ }^{11}$, qui représente aujourd'hui le premier budget communautaire. Parce qu'elle intéresse tout spécialement la compétitivité économique et l'indépendance énergétique de l'Union, la filière forêt-bois est susceptible de trouver un nouvel élan à l'échelle européenne.

Un relais ne s'établit-il pas entre la dynamique politique communautaire qui s'enclenche et un « moteur » forestier national qui s'essouffle?

\section{B - La place de l'Etat forestier est implicitement remise en cause}

Surdéterminée par les orientations économiques et environnementales tracées au niveau européen, la politique forestière nationale paraît manquer de souffle. A la faiblesse conceptuelle s'ajoute l'érosion des moyens financiers d'action, tant en forêt publique qu'en forêt privée. Hors de l'hexagone, plusieurs Etats-membres de l'Union ont fait le choix de décentraliser la gestion forestière au niveau régional.

\section{Quelle plus-value pour la politique forestière nationale?}

Avec l'émergence d'une politique forestière européenne, appuyée sur des orientations stratégiques claires et dotée d'une assez large gamme d'instruments financiers, se pose naturellement la question de la place qui revient au cadre national hérité de l'administration des Eaux et Forêts. L'interrogation est d'autant plus forte que les concepts soutenant la politique forestière nationale paraissent largement redondants avec ceux portés par le niveau européen et que les moyens dont dispose l'Etat forestier vont en décroissant.

Le diagnostic et le plan d'action contenus dans le Programme forestier national (PFN) pour la période 2006-2015 ont été définis en 2006 par le ministère de l'agriculture sur la base des propositions formulées par le Conseil supérieur de la forêt, des produits forestiers et de la transformation du bois. Ils ne renouvellent pas la réflexion et les perspectives sur la gestion de l'espace forestier. Reflet d'une position consensuelle alimentée par la prise de conscience internationale sur la nécessité de lutter contre le changement climatique global et l'érosion de la biodiversité tout en conservant la fonction récréative des espaces forestiers, le PFN traduit à l'échelle nationale les orientations déjà fixées par l'Union européenne sans apporter une véritable plus-value.

Sur le plan financier, la stratégie nationale avancée pâtit par ailleurs d'un manque évident de moyen et, par conséquent, de crédibilité. Depuis le rapport Bianco de 1998 "La forêt, une chance pour la France $~^{12}$ et en dépit des incantations répétées en faveur d'un réinvestissement dans la filière forêt-bois, force est de constater que la surface 
financière de l'Etat forestier s'effrite. La subvention du budget de l'Etat destinée à la mise en oeuvre du régime forestier par l'ONF sous le titre de versement compensateur s'érode. Difficile à percevoir compte tenu de l'aide exceptionnelle consentie par l'Etat pour la reconstruction des forêts publiques après les tempêtes de $1999^{13}$, la diminution de la contribution nationale à la gestion des forêts soumises au régime forestier est pourtant bien réelle. Dans le cadre du contrat d'objectif liant l'Etat à l'ONF sur la période 2001-2006, le versement compensateur doit en effet s'établir autour de $122 \mathrm{M} €$ en $2006^{14}$. Il atteignait $133 \mathrm{M} €$ en $1999^{15}$. Pour les forêts communales et privées, la suppression du FFN en 2000 marque un tournant majeur (Buttoud, 2002). Ce fonds spécial du trésor, devenu archaïque dans le système budgétaire redéfini par la Loi organique sur les lois de finances du $1^{\mathrm{er}}$ août 2001 (LOLF), disparaît et avec lui une contribution essentielle à l'investissement en forêt communale et privée.

Le PFN note le poids croissant de l'Union européenne et des collectivités territoriales dans le financement de la politique forestière sans pour autant reconnaitre la place éminente qui leur revient (pour l'Union) ou qui devrait leur revenir (pour les conseils régionaux) dans l'élaboration de cadre de référence stratégique. Le PFN note ainsi qu' " Une grande partie des financements forestiers relève de cofinancement par les collectivités dans le cadre des contrats de plan Etat-Région qui ont été des vecteurs privilégiés. " Le Programme forestier national ajoute que "Les financements européens ont pris une importance particulière avec le règlement de développement rural 2000-2006. " Implicitement, le PFN exprime le retrait de l'Etat dans le financement de la politique forestière.

En termes de cohérence administrative et financière, remarquons, en outre, que le fait de définir un PFN sur dix ans (2006-2015) alors que l'Etat ne dispose d'aucun cadre budgétaire pluriannuel ne contribue guère à la crédibilité de sa politique forestière. En contrepoint, la définition d'une politique forestière européenne calée sur la durée des perspectives financières pluriannuelles de l'UE (2007-2012) renvoie l'image d'un cadre stratégique et opérationnel compact, susceptible d'être réellement mis en œuvre et par conséquent suivi d'effets.

19 Au sein de l'Union, plusieurs Etats-membres ont d'ailleurs commencé de mettre leur politique forestière à l'heure européenne, si ce n'est avec enthousiasme et facilité, du moins avec réalisme et résolution.

\section{Le chemin de la décentralisation suivi par plusieurs états-membres}

Grâce au travail pionnier des géographes Paul Arnould, Micheline Hotyat et Laurent Simon sur "Les forêts d'Europe", nous disposons d'une vue assez large et indépendante sur le positionnement des politiques forestières dans le champ institutionnel. Daté de 1997, l'ouvrage montre que de nombreux états membres de l'Union européenne ont régionalisé leur politique forestière en décentralisant le pilotage de la gestion sylvicole. Les collectivités régionales élues - et non pas les services déconcentrés de l'Etat central - se sont vu confier la responsabilité forestière.

21 En Italie, la gestion des forêts est passée sous la responsabilité des régions depuis 1977 sur la base d'une orientation constitutionnelle déjà posée par la Constitution de 1947. Les collectivités bénéficient d'une large compétence dont le fondement ne se trouve pas seulement dans la cohérence à donner au développement économique territorial mais également dans l'harmonisation des objectifs de production de bois et de préservation de la nature. L'ouvrage de 1997 souligne que le transfert de la compétence « forêt » de 
l'Etat central aux régions ne s'est pas fait sans mal. Le repositionnement de l'ancienne administration des gardes forestiers piémontais apparait encore comme une question en suspens. En Espagne, sous l'impulsion de la Constitution de 1978, les communitades autonomias ont également gagné la compétence de gestion forestière. La aussi, la reconversion de l'administration forestière préexistante - l'Icona - est apparue comme une contrainte forte à gérer dans la durée. Par comparaison aux deux exemples développés, la France paraît à la traîne du courant de décentralisation. Sans doute l'analyse comparée de l'histoire politique générale de l'Italie, de l'Espagne et de la France apporte-t-elle un bémol à cette observation. Au sortir de la période fasciste pour l'Italie et de l'ère franquiste pour l'Espagne, la décentralisation, y compris en matière forestière, apparait en effet comme une réaction naturelle et une précaution contre le pouvoir central. Il convient, en toute rigueur, de considérer l'évolution de politique forestière dans un contexte national assez apaisé afin d'évaluer la plus ou moins grande résilience du modèle centralisateur français.

Vue sous cet angle, l'analyse de l'évolution du cadre de la politique britannique apporte un éclairage complémentaire qui conforte la tendance soulignée en faveur d'un accroissement des pouvoirs locaux dans le secteur forestier. L'exemple offert par le Royaume-Uni est particulièrement significatif de la puissance du mouvement amorcé dans la mesure où il s'applique à un pays de longue tradition démocratique et centralisatrice qui n'a reconnu que très tardivement le fait régional au sein de la common law. Complexe, très variable dans ses fondements juridiques et dans sa portée selon les territoires composant le Royaume, le processus de devolution - développé à partir de 1998 - transfert toutefois systématiquement les compétences de l'Etat central en matière forestière aux gouvernements d'Ecosse, d'Irlande du Nord ou du Pays de Galles. Portés par une logique qui privilégie la définition de politique de développement économique et d'aménagement du territoire au plus près du terrain, le Scotland Act, le Northern Ireland Act et le Wales Act, donnent à la politique forestière une assise régionale ${ }^{16}$. S'adaptant aux évolutions politiques, non sans difficulté administrative, la Forestry Commission, qui assurait jusque-là une certaine cohérence à la politique forestière britannique, est scindée. Un comité des commissaires Board of Commissioners continue de réunir les commissions forestières régionales (Forestry commission England, Forestry Commission Wales, ...) afin de préserver un cadre de réflexion commun et de mutualisation des expériences. La recherche forestière reste confiée à une agence britannique - Forest Research - qui a une fonction de veille et d'innovation au profit de l'ensemble des politiques forestières du Royaume-Uni.

Après les évolutions enregistrées dans plusieurs pays d'Europe au cours des années 70, les changements intervenus en Grande-Bretagne depuis dix ans confirment la tendance à la régionalisation des politiques forestières et interroge sur le positionnement singulier de la France. L'interrogation apparaît d'autant plus forte que dans l'hexagone aussi, les régions - c'est-à-dire les conseils régionaux, assemblées élues - s'affirment comme les assembliers du développement économique et de l'aménagement du territoire, dont la forêt est un élément. 


\section{II - La Région apparaît comme le nouveau pivot institutionnel de la mise en œuvre de la politique forestière}

\section{A - Depuis 1985, le niveau régional est déjà reconnu comme étant le plus pertinent pour la mise en œuvre d'une politique forestière opérationnelle}

Actuellement, la gouvernance forestière territoriale s'appuie sur l'échelon régional déconcentré de l'Etat. Assez globales, les orientations forestières définies à ce niveau ne se déclinent pas de façon spécifique pour les forêts publiques, singulièrement pour les bois communaux. Les Communes forestières appellent à une meilleure prise en compte de leur aspiration et à un renforcement de la présence des conseils régionaux dans l'élaboration de politiques forestières adaptées aux réalités et aux besoins des territoires.

\section{La politique forestière française : une politique centralisée et déconcentrée}

Elaborée un peu après les grandes lois de décentralisation du début des années quatrevingt, la loi du 4 décembre 1985 d'orientation sur la forêt ne rompt pas avec la tradition très centralisatrice de la politique forestière française mais introduit, pour la première fois dans ce champ d'action publique, des éléments de déconcentration de la prise de décision nationale. Le mouvement amorcé reste cependant timide eu égard à la diversité des situations foncières et naturelles qui fondent la variabilité intrinsèque de la forêt française ${ }^{17}$.

26 La loi d'orientation forestière du 9 juillet 2001 abroge les dispositions de la loi de 1985 sans modifier l'économie générale du dispositif de pilotage stratégique de la gestion des forêts. En l'état actuel du droit, le code forestier campe sur un mode de gouvernance territoriale placé sous l'autorité du préfet de région, auquel est adjoint une commission régionale de la forêt et des produits forestiers, à vocation consultative. Dans ce cadre, des orientations régionales forestières sont arrêtées. De portée assez générale, elles fixent les grandes orientations de gestion pour l'ensemble des forêts privées et publiques, domaniales ou communales, de la région.

\section{Le cadre différencié de la gestion des forêts publiques et des forêts privées}

27 La forêt privée bénéficie d'une déclinaison opérationnelle des ORF à travers le schéma régional de la forêt privée. Ce document permet de convertir les orientations générales au cas spécifique de la sylviculture privée qui doit nécessairement intégrer les contraintes économiques de court et moyen terme pesant sur les propriétaires particuliers ou institutionnels.

28 Afin de mettre en œuvre le schéma régional, les centres régionaux de la propriété forestière (CRPF) assurent une mission d'animation et d'information des propriétaires privés. Ils veillent au respect des grandes priorités forestières définies sur leur territoire en examinant chacun des plans simples de gestion ${ }^{18}$ (PSG). En droit, les CRPF sont des établissements publics d'Etat à caractère administratif (EPA). En fait, nonobstant la présence d'un commissaire du gouvernement censé représenter l'Etat, la 
composition de leur organe dirigeant fait la part belle aux représentants des propriétaires privées régionaux. L'action des CRPF s'inscrit en réalité dans une logique de décentralisation fonctionnelle régionale de la politique des forêts privées.

Par contraste, les forêts publiques ne bénéficient pas d'une stratégie régionale propre établie en étroite concertation avec les élus locaux, représentants des communes forestières et moins encore du conseil régional. Cette absence de déclinaison légale ou réglementaire des $\mathrm{ORF}$ aux cas des forêts domaniales et surtout des bois communaux n'exclut évidemment pas l'instauration de bonnes relations de travail entre les collectivités et l'Etat. Elle reste cependant peu compatible avec l'esprit et la lettre du principe constitutionnel de libre administration des collectivités territoriales et semble à contre-courant du souffle de la décentralisation. Selon les termes de l'article 72-1 de la Constitution, « Dans les conditions prévues par la loi, ces collectivités s'administrent librement par des conseils élus et disposent d'un pouvoir réglementaire pour l'exercice de leurs compétences ». L'encadrement légal de la gestion de la propriété forestière communale trouve sa source dans les dispositions de l'article L.111-1 du code forestier ${ }^{19}$.

Donnant corps à l'autorité du régime forestier sur les bois communaux, l'Office national des forêts (ONF) assure la gestion de l'ensemble des forêts publiques sans s'appuyer sur une stratégie régionale différenciée de celle des forêts privées, plus fine que celle posée par les ORF et accessible aux citoyens eu égard à la nécessaire transparence de la gestion publique. Etablissement public de l'Etat à caractère industriel et commercial (EPIC), l'Office ne structure d'ailleurs pas son organisation territoriale en se calant sur la trame régionale (cf. Carte A). Depuis la fin des années quatre-vingt-dix, l'opérateur forestier de l'Etat a développé un maillage tout à fait spécifique de directions territoriales, le plus souvent interrégionales, ce qui rend difficile, sur le plan administratif, le passage d'une stratégie d'établissement pensée à l'échelle nationale vers une réflexion régionalisée menée avec des élus locaux. 


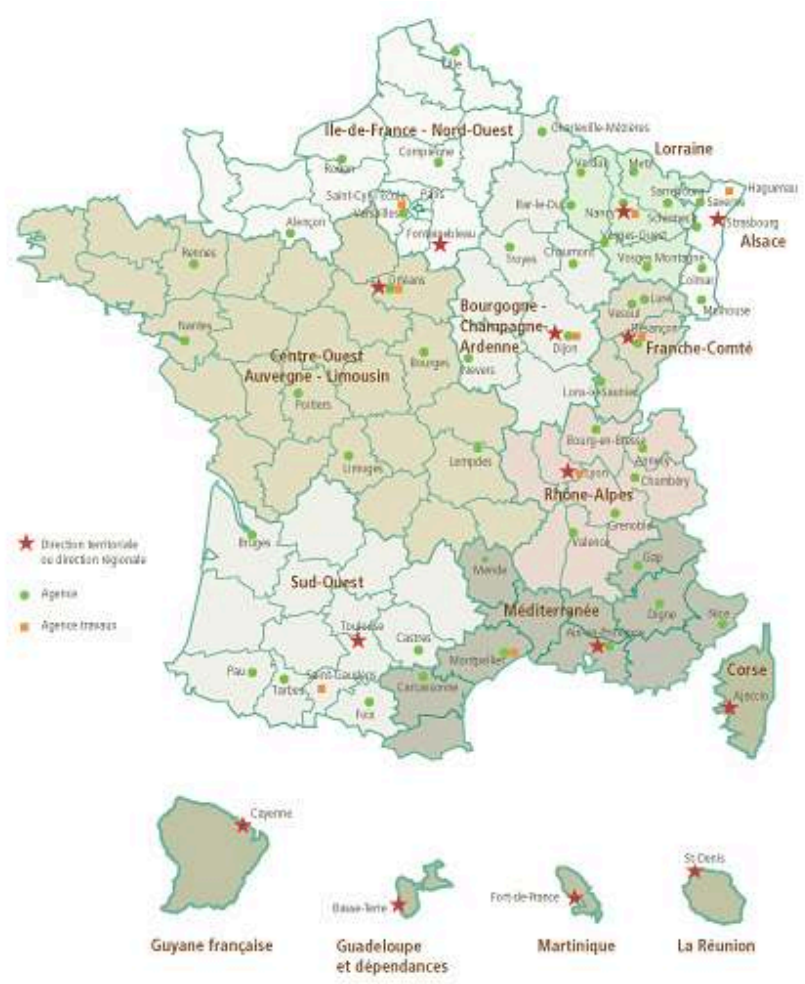

31 L'attente des élus locaux demeure, il est vrai, ambigüe. Une lecture rapide du rapport établi en 2003 par Jean-Claude Monin pour le compte des Communes forestières ${ }^{20}$ peut donner l'impression d'un attachement des collectivités territoriales au système en place. Celui-ci est fondé sur le respect du régime forestier et sur la position monopolistique de l'ONF comme sylviculteur public ; il semble perçu comme un système «qui marche ». Toutefois, dans le détail, le ton du rapport et plusieurs de ses propositions clés accréditent l'idée que les Communes forestières souhaiteraient voir accorder une plus large place aux collectivités territoriales, en particulier aux Régions, dans la gouvernance forestière. La déclinaison des ORF en stratégies opérationnelles régionales définies sous l'autorité d'élus est promue. Tout en considérant avec intérêt le développement des chartes forestières de territoires, établies à l'échelle d'un massif sylvicole donné, le rapport Monin note cependant que ce type d'outil reste limité par l'espace intercommunal qui l'enserre. Les Communes forestières appellent en réalité à une réflexion portée par des élus au niveau régional, c'est-à-dire du conseil régional. S'agissant de l'office et de son repositionnement géopolitique vis-à-vis de l'affirmation des collectivités territoriales, le rapport Monin, sous couvert d'un apparent respect de l'opérateur forestier de l'Etat, n'est pas tendre. Avec un certain humour, il met l'ONF sous pression de s'adapter aux temps nouveaux de la décentralisation: "L'Office national des forêt fait souvent l'objet de métaphores maritimes : de "navire amiral" de la forêt française $e^{21}$, figure de proue de la politique forestière, au "super-pétrolier", structure géante aux manoeuvres difficiles. Aujourd'hui, l'ONF ne peut rester à l'écart des évolutions présentés tout au long de ce rapport, au risque de se transformer, à terme, en "vaisseau fantôme". "

Le rapport Monin marque un tournant dans l'appréhension par les Communes forestières du régime forestier et de son opérateur historique. Cette évolution s'inscrit, à vrai dire, dans le courant puissant de décentralisation des politiques de 
développement économiques et d'aménagement du territoire, lancé depuis le début des années 80 en France.

\section{B - Les conseils régionaux s'imposent comme les nouveaux organisateurs de l'aménagement du territoire et du développement économique durable}

33 L'affirmation de la place des conseils régionaux dans la définition de politiques forestières infranationales est cohérente avec la montée en puissance de leurs prérogatives en matière d'aménagement du territoire et de développement économique. La loi d'août 2004 relative aux libertés et responsabilités locales marque de ce point de vue une étape majeure. En Corse, la loi de janvier 2002 a déjà amorcé le mouvement d'une large décentralisation de la politique forestière et a permis de reconsidérer les rapports de la Collectivité territoriale de Corse avec l'Etat forestier et son opérateur.

\section{La tendance lourde en faveur de l'affirmation du rôle des Régions depuis 25 ans}

34 Le rôle clef que jouent les conseils régionaux dans le domaine du développement économique ne date pas d'aujourd'hui (Bodineau et Verpeaux, 1993). Dotées, dès la loi du 7 janvier 1983 relative à la répartition des compétences entre les communes, les départements, les régions et l'Etat, d'une compétence d'attribution en la matière, les Régions ont vu cependant croître leurs prérogatives dans la seconde moitié des années 90 et au début de la décennie suivante. Elles ont en particulier une mission de planification et de coordination des acteurs économiques territoriaux.

Le rôle des conseils régionaux en matière d'aménagement du territoire progresse à partir de 1995 avec la loi du 4 février 1995 d'orientation pour l'aménagement et le développement $\mathrm{du}$ territoire (LOADT). Celle-ci instaure le schéma régional d'aménagement et de développement du territoire (SRADT) comme outil de planification régional. Perfectionné par la loi d'orientation pour l'aménagement et le développement durable du territoire (LOADDT) du 25 juin 1999 qui le rend plus opérationnel, le SRADT fixe les orientations fondamentales à moyen terme, de développement durable du territoire régional. Il veille à la cohérence des projets d'équipement avec la politique de l'Etat et des différentes collectivités territoriales. Le schéma régional se traduit par un document d'analyse prospective - incluant éventuellement une vision interrégionale ou transfrontalière -, une charte d'aménagement et de développement durable du territoire régional et des documents cartographiques - expressions concrètes et spatialisées des orientations et des options retenues par le Conseil régional en concertation avec les parties prenantes (conseil économique et social régional, acteurs socio-professionnels, conseils généraux, ...).

L'acte II de la décentralisation porté par la loi du 13 août 2004 relative aux libertés et responsabilités locales est souvent perçu comme un demi-succès. Il marque pourtant une avancée substantielle - qui reste encore à prolonger, notamment en matière de gestion des fonds structurels (Degron, 2007) - dans la reconnaissance du rôle de premier plan joué par les conseils régionaux en matière de développement économique (Benoît, 2005). L'article $1^{\text {er }}$ de la loi offre la possibilité aux Régions d'élaborer, dans le cadre d'un processus d'expérimentation, des schémas régionaux de développement 
économique (SRDE). Le cadre de l'expérimentation est largement étendu par la décision du Conseil constitutionnel du 12 août 2004 (DC n²004-503, Loi relative aux libertés et responsabilités locales). En pratique, tous les conseils régionaux métropolitains sont aujourd'hui dotés ou en passe de se doter de cet outil qui permet une mise en cohérence globale des actions jusque-là dispersées des collectivités régionales en matière d'aménagement du territoire, d'aides économiques et de formation professionnelle.

37 Les déclinaisons sectorielles des SRDE sont encore très variables d'une région à l'autre. La plupart des schémas ont une vocation généraliste et survolent les problématiques économiques territoriales plus qu'ils ne définissent une véritable feuille de route opérationnelle. Toutefois, certains SRDE montrent la voie d'une réelle prise en main, par les conseils régionaux, d'axes stratégiques de développement et des outils pour les mettre en oeuvre. Ainsi, à titre d'exemple, le SRDE de la région Centre est-il suivi par une série de schémas sectoriels dont une Stratégie régionale de développement agricole et forestier (SRDAF) et un Schéma régional de développement touristique (SRDT) qui convergent pour tendre vers une meilleure valorisation du patrimoine naturel $\mathrm{du}$ Centre, en particulier de ses forêts ${ }^{22}$.

Partant de ce type d'approche, on conçoit que le pas à franchir pour que les Régions deviennent les donneurs d'ordre d'une politique forestière plurielle, au diapason de la diversité des territoires de la Nation, ne soit plus très difficile à franchir.

\section{Le cas de l'Assemblée territoriale de Corse : cas particulier ou point de convergence?}

39 Le pas est franchi en milieu méditerranéen et dans le contexte politique corse où l'Assemblée territoriale reçoit une large délégation de compétence en matière de gestion forestière ${ }^{23}$. La loi du 22 janvier 2002 relative à la Corse transfert le domaine forestier privé de l'État (environ 55000 hectares) à la Collectivité territoriale de Corse (CTC). Celle-ci en assure la gestion conformément au code forestier dont le régime est préservé mais dont les conditions pratiques de mise en œuvre s'adaptent au territoire. Si l'état boisé est toujours protégé de par la loi, le changement de propriétaire implique, forcément, une révision de la logique publique d'aménagement forestier. Le transfert en pleine propriété des forêts de l'Etat à la CTC est devenu effectif le 1er janvier 2004. La CTC s'est alors engagée dans la définition d'orientations générales de la politique forestière qui ont fait l'objet d'un débat à l'Assemblée de Corse le 23 octobre 2006. Ces orientations servent de base à l'élaboration des orientations forestières en partenariat avec l'Etat et les acteurs de la filière forêt-bois pour la période 2007-2013. Elles seront intégrées au Plan d'aménagement et de développement durable de la Corse (PADDUC) qui doit se substituer au Plan de développement de la Corse et au Schéma d'aménagement de la Corse actuels.Notons que la période couverte par les ORF Corse, elles-mêmes fondées sur les orientations générales de la politique forestière établies par la CTC, correspond à la temporalité des fonds structurels européens et du contrat de projet Etat-Région : décentralisation et réalisme financier vont de paire.

40 En pratique, les priorités affichées par la CTC rejoignent les items traditionnels de la gestion forestière durable et multifonctionnelle mais, en s'appuyant sur la volonté exprimée par la CTC, elles profitent pleinement des outils multiples à disposition de l'Assemblée territoriale pour accompagner une mise en œuvre opérationnelle optimale. Ainsi, les premières priorités corses visant à «optimiser la mobilisation du bois d'œuvre : 
améliorer les réseaux de desserte des forêts productives » et à développer l'usage du "bois énergie : mobiliser tous les acteurs de la filière" s'appuient naturellement sur les orientations du SRADT et sur la compétence économique générale de l'Assemblée territoriale. Après l'affirmation du renforcement de la protection des forêts contre l'incendie et du nécessaire maintien de la biodiversité, qui participent à la qualité de vie et à l'attractivité de l'île de beauté, la CTC se positionne en faveur d'une professionnalisation de l'accueil du public en forêt. Là encore, la logique de développement économique et touristique est sous-jacente. Elle paraît d'autant plus pertinente et réaliste que l'Assemblée territoriale dispose du méta outil de formation que représente le Plan régional de développement des formations professionnelles (PRDF) dont le spectre est étendu à l'ensemble des publics, jeunes ou adultes, de la formation professionnelle initiale ou continue par la loi du 17 janvier 2002 de modernisation sociale.

41 Sur le plan organique, la Corse se félicite de la création récente d'un Centre régional de la propriété forestière (CRPF) destiné à favoriser la mise en valeur des forêts privées insulaires. S'agissant de l'office national des forêts, la CTC note que dans les forêts domaniales et les forêts soumises au régime forestier, les conditions d'une gestion durable, entre exploitation et préservation de la ressource future, semblent réunies dans le cadre d'une mise en valeur raisonnée et respectueuse de l'environnement. Sur la base de ce constat, la CTC confie donc à l'ONF une délégation de service public de gestion forestière. Un contrat de 12 ans est signé entre la CTC et l'ONF selon la règle de la régie intéressée; une partie de la rémunération du délégataire est fixe, l'autre correspond à $50 \%$ des recettes. Il est important de souligner que le rôle de l'office ne tient plus, en Corse, à la seule invocation de la soumission des forêts publiques au régime forestier mais, également, à la perception globalement positive qu'ont des élus territoriaux de l'aménagement et de la sylviculture pratiqués par le principal opérateur forestier local. Celui-ci est d'ailleurs susceptible d'être soumis au principe de libre concurrence. En Corse, dans une certaine mesure, les rapports entre les élus et les techniciens s'équilibrent : les choix décisifs pesant sur l'évolution de l'espace forestier régional dépendent dorénavant d'élus, dans un cadre juridique d'exception, qui oblige les techniciens forestiers à revoir leur pratique et à maintenir une qualité optimale de services et de travaux sylvicoles. A la légitimité politique répond une légitimité technique. Tout en étant chacune respectable, les deux légitimités demeurent naturellement hiérarchisées.

Comme Jean-Jacques Rousseau l'avait déjà remarqué à travers son projet de constitution pour la Corse de 1763, l'île de beauté apparait comme un laboratoire de la démocratie (Rousseau, réed. 2000). L'expérience forestière qui s'y mène doit-elle inspirer la réforme d'une politique historiquement centralisée ? Plus que jamais, l'espace de la politique forestière française est en question.

\section{Conclusion}

43 Ancrée dans un cadre institutionnel et une culture administrative très centralisée, la politique forestière française paraît néanmoins devoir évoluer sous la double influence de la construction européenne et de la décentralisation.

Difficiles à percevoir, les changements qui s'opèrent depuis le début des années 90 se révèlent nettement sous le prisme d'une analyse géographique multiscalaire qui 
combine les dimensions européenne, nationale et régionale du fait forestier et qui replace celui-ci dans une perspective élargie de développement économique durable.

De ce point de vue, le concept de géoalliance paraît offrir une grille de lecture originale et utile à la perception de l'espace renouvelé des politiques publiques, notamment dans le domaine forestier.

\section{BIBLIOGRAPHIE}

Arnould p., Hotyat M. et Simon L. (1997) - Les forêts d'Europe, Paris, Nathan, 413 p.

Benoit L. (2005) -Décentralisation et développement économique : la nouvelle répartition des compétences, Paris, Actualité juridique du droit administratif,10 octobre 2005.

Bodineau p. et Verpeaux M. (1993) Histoire de la décentralisation, Paris, Presses universitaires de France, $125 \mathrm{p}$.

Buttoud G., 1983. L'Etat forestier - Politique et Administration des forêts dans l'histoire française contemporaine, université de Nancy 2, thèse d'Etat en économie-politique, 1983, 691 p.

Buttoud G., La politique forestière française, du rapport Bianco à la loi de juillet 2001, Berne, Schweizerische Zeitschrift fur Forstwesen, vol. 153, 2002/8.

Collectif (1987) - Les Eaux et Forêts du 12ème au 20ème siècle, Paris, C.N.R.S., 767 p.

Corvol A., Arnould p. et Hotyat M. (sous la direction de) (1997) - La forêt : Perceptions et représentations, Paris, Group.Hist.For.Fr., L'Harmattan, 401 p.

Degron R. (1998) - La conversion des forêts domaniales françaises (1860-1888) : une grande vague brisée, Nancy, Rev.For.Fr., 1, p. 71-84.

Degron R. (1999) - Forêts, Temps et Sociétés - Les conversions forestières de Lorraine : bilan, contrastes, rythmes et ruptures, thèse de doctorat en géographie à l'université de Nancy 2 sous la direction de Jean-Pierre Husson, 1999, 404 p.

Degron R. (2007) - La décentralisation de la gestion des fonds structurels européens : une expérimentation au milieu du gué, Paris, Actualité juridique du droit administratif, 30 avril 2007.

Degron R. (2007) - Etat, départements et communes : une géoalliance sociale exclusive, Paris, revue Pouvoirs locaux, $\mathrm{n}^{\circ} 75, \mathrm{IV} / 2007$, décembre 2007.

Degron R. (2008) - L’Union européenne, les régions, les agglomérations et les pays :

l'administration territoriale de l'Europe face à l'Économie-Monde, Paris, revue Pouvoirs locaux, $\mathrm{n}^{\circ} 77$.

Eurofor. (1994) - L'Europe de la forêt, Luxembourg, Ed. Parlement européen, 2 tomes, 1528 p.

Rousseau J.J. (1763) - Projet de constitution pour la Corse, Paris, Ed. Robert Chesnais - Nautilus, 2000.

Vidal de la Blache p. (1917) - La France de l'Est (Lorraine-Alsace), Paris, $2^{\circ}$ édition de 1994 avec préface d'Y.Lacoste, Collection La Découverte, 286 p. 
Vidal de la Blache p. (1903) - Tableau géographique de la France, Paris, La Table Ronde, Edition de 1994, 559 p.

\section{NOTES}

1. Quelque peu diluée dans le «Tableau géographique de la France », la forêt tient en revanche une place centrale dans l'analyse développée par Paul Vidal de la Blache dans « La France de l'Est (Lorraine-Alsace) ».

2. L'approche du fait forestier au sein du Groupe d'histoire des forêts françaises est révélatrice de la richesse du fait forestier et de la nécessaire mobilisation interdisciplinaire qu'il impose, avec notamment une ouverture vers les sciences sociales (cf. en particulier sur ce point, Corvol A., Arnould p. et Hotyat M. (sous la direction de), 1997. La forêt : Perceptions et représentations).

3. A l'époque, la direction générale des Eaux et Forêts était rattachée au département ministériel des finances. Le passage à l'agriculture date seulement de 1886 .

4. Avant l'élargissement de 1996, le taux de boisement de l'UE 12 était de 28,8\%. Après l'intégration de la Finlande (taux de boisement de 73,9\%), de la Suède $(66,9 \%)$, de l'Autriche $(46,7 \%)$, le taux moyen de boisement de l'UE 15 passe à 35,1\% (données FAO, 2006).

5. En Allemagne, $47,3 \%$ de la forêt est privée, 29,6\% appartient au domaine des Länder, 19,5\% correspond aux forêts des collectivités infra lander. La Fédération ne possède en propre que 3,7\% du patrimoine sylvicole du pays (données Inventaire forestier fédéral, enquête 2001-2002).

6. L'instrument financier pour l'environnement (Life)

7. Communication de la Commission au Conseil et au Parlement européen - Rapport sur la mise en oeuvre de la stratégie forestière de l’Union européenne, CoM (2005) 84 (final) du 10 mars 2005

8. Communication de la Commission au Conseil et au Parlement sur le plan d'action forestier de l'UE, COM (2006) 302 (final) du 15 juin 2006

9. Fonds européen agricole pour le développement rural (FEADER)

10. Fonds européen d'orientation et de garantie agricole (FEOGA)

11. Fonds européen de développement régional (FEDER)

12. Rapport de M. Jean-Louis Bianco, «La forêt, une chance pour la France ", réalisé à la demande de M. le Premier ministre, 25 août 1998 (http://www.ladocumentationfrancaise.fr/ rapports-ublics/994000095)

13. Source: Programme annuel de performance $n^{\circ} 149$ «Forêt», annexe au projet de loi de finances pour 2006

14. Source : Ministère de l'agriculture, Les dossiers techniques du ministre - Le contrat Etat-ONF 2001-2006, 2001

15. Source : Sénat, Rapport général de la commission des finances sur le projet de loi de finances initiale pour 1999, 1998

16. Cf. House of Commons, An introduction to devolution in the UK, Research paper 03/83, 2003

17. Voir sur ce point le rapport Monin (p. 8) qui dresse une fiche d'identité de la forêt française synthétique et richement illustrée de nombreuses cartes. Sur le plan de la diversité écologique, on pourra se reporter au travail réalisé par l'Inventaire forestier national (IFN), lui aussi appuyé sur de nombreuses cartes ainsi que sur une utilisation originale et spatialisée de l'indice de Shannon.

18. Le Plan simple de gestion est le document d'aménagement forestier qui doit être établi pour les forêts privées dont la surface dépasse 25 ha d'un seul tenant.

19. L'article L. 111-1 du code forestier dispose que "Relèvent du régime forestier et sont administrés conformément aux dispositions du présent livre ... $2^{\circ}$ Les bois et forêts susceptibles 
d'aménagement, d'exploitation régulière ou de reconstitution et les terrains à boiser mentionnés à l'article L. 141-1, appartenant aux régions, aux départements, aux communes... ».

20. Rapport «Décentralisation et politique forestière : propositions pour les communes forestières ", Jean-Claude Monin, Février 2003

21. Le terme est employé par Jean-Louis Bianco dans son rapport « La Forêt, une chance pour la France ».

22. Avec 870000 ha de forêt, la région Centre est la 6ème région forestière française par la taille. Les formations boisées régionales sont principalement composées de chênes sessiles et pédonculés ainsi que de pins. La forêt est ici formée à $85 \%$ de propriétés privées, pour l'essentiel en Sologne. Du côté de la forêt publique, plusieurs grands massifs domaniaux, issus de la liste civile des Orléans, sont à signaler, en particulier autour de la capitale régionale, avec une fonction d'accueil périurbain marquée.

23. La nature corse produit une forêt d'essence variée, dont les équilibres sont bien différents de ceux de la forêt française métropolitaine. Sur les 238000 ha de forêt corse, près de $52 \%$, soit environ 123000 ha, sont constitués de peuplement de chêne vert dominant. La propriété de la forêt corse est aujourd'hui répartie entre la Collectivité territoriale corse (23\% de la surface forestière), les autres collectivités territoriales, pour l'essentiel les communes (32\%) et les privés (45\%).

\section{RÉSUMÉS}

De longue date, la gestion forestière française procède de la mise en œuvre d'une politique nationale fortement centralisée. Aujourd'hui, celle-ci paraît susceptible d'évoluer dans un cadre spatial ouvert à la dimension européenne et au fait régional. La récente adoption d'une politique forestière communautaire concerne la période 2007-2011. Elle définit de claires priorités d'action et des moyens financiers étendus, ce qui remet implicitement en cause la pertinence d'une stratégie forestière strictement nationale. Les objectifs de celle-ci recoupent en effet largement ceux fixés par l'Union; ses moyens sont, par ailleurs, assez modestes. Dans le même temps, l'affirmation des conseils régionaux comme assembliers du développement économique et de l'aménagement $d u$ territoire interroge sur la permanence d'une gouvernance sylvicole infra nationale uniquement fondée sur l'échelon déconcentré régional de l'Etat. L'absence de déclinaison des orientations régionales forestières au cas particulier de la gestion des forêts publiques, spécialement communales, tranche avec le cadre sur mesure dont bénéficient les forêts privées. La maturation des politiques économique régionales, y compris dans leur volet sectoriel forestier, laisse entrevoir la possibilité d'une prise en main par les élus locaux de la destinée des forêts de leur territoire. Le cas corse illustre une voie d'évolution décentralisée du cadre institutionnel de la politique forestière française. Au sein de l'Union, plusieurs Etatsmembres voisins inscrivent déjà la gestion sylvicole dans une logique décentralisatrice.

From a long time, France implements a strong national forest policy. However, the European and regional levels have to play a growing role in this field of public policy. Recent adoption of an European forest policy for 2007-2011, with clear priorities and wide financial means, takes a new look on pertinence of a single national forest strategy. Meanwhile, the rising of conseils régionaux as coordinators for economic and territory development calls into question the French national forest governance framework. To a certain extend, the Corsican case shows a way for a true 
politic devolution of forest policy. Within the European Union, many member-states have already chosen this kind of approach.

INDEX

Keywords : forest, forest policy, Europe, France, devolution

Mots-clés : forêt, politique forestière, Europe, France, décentralisation

\section{AUTEUR}

\section{ROBIN DEGRON}

Chercheur associé au LERAD (EA 2108)UFR Droit, Economie et Sciences socialesUniversité François Rabelais de Tours50 avenue Jean Portalis - 37200 TOURSCourriel : robin.degron/CHEZ/ voila.fr 